\title{
STATISTICAL PROPERTIES OF ENDOMORPHISMS AND COMPACT GROUP EXTENSIONS
}

\author{
IAN MELBOURNE AND MATTHEW NICOL
}

\begin{abstract}
The statistical properties of endomorphisms under the assumption that the associated PerronFrobenius operator is quasicompact are considered. In particular, the central limit theorem, weak invariance principle and law of the iterated logarithm for sufficiently regular observations are examined. The approach clarifies the role of the usual assumptions of ergodicity, weak mixing, and exactness.

Sufficient conditions are given for quasicompactness of the Perron-Frobenius operator to lift to the corresponding equivariant operator on a compact group extension of the base. This leads to statistical limit theorems for equivariant observations on compact group extensions.

Examples considered include compact group extensions of piecewise uniformly expanding maps (for example Lasota-Yorke maps), and subshifts of finite type, as well as systems that are nonuniformly expanding or nonuniformly hyperbolic.
\end{abstract}

\section{Introduction}

It has been well known since the work of Sinai, Ruelle and Bowen (see for example [8, 31, 33]) that mixing uniformly expanding and uniformly hyperbolic dynamical systems enjoy strong statistical properties, such as exponential decay of correlations and the central limit theorem, for sufficiently regular observations. Since then, a great deal of effort has been made to extend these results to more general classes of dynamical systems and observations (see, for example, the recent surveys in $[\mathbf{1}$, 2, 35]).

One approach is to study the rate of decay of certain transfer operators or PerronFrobenius operators when restricted to suitable function spaces. In many important cases, it is possible to prove that these operators are quasicompact, leading to exponential decay rates. Exponential decay of correlations follows immediately, while the central limit theorem follows from an idea of Gordin [17]. A functional version of the central limit theorem is also known to hold in such situations, and it was recently noticed [16] that the upper half of the law of the iterated logarithm is valid (provided that the relevant function space can be chosen to lie in $L^{\infty}$ ).

In a different direction, [16] considered compact group extensions of uniformly hyperbolic diffeomorphisms and, the authors being motivated by [28], restricted to a class of equivariant observations. Using a combination of existing techniques and new ideas, it was shown that (improved versions of) the statistical properties described above are inherited by the group extension. In particular, we note the improved results on nondegeneracy of the central limit theorem in $[\mathbf{1 6}]$.

In this paper, we describe new results in the theory of statistical properties of (a) dynamical systems, and (b) their compact group extensions. In direction (a), we give an account of the implications of the quasicompactness of the Perron-Frobenius

Received 24 October 2002; revised 25 March 2003.

2000 Mathematics Subject Classification 37C30 (primary), 37A50, 60F05, 37C80 (secondary). 
operator for the statistical behaviour of endomorphisms. Our approach clarifies the usual assumptions of ergodicity, weak mixing, and exactness. In particular, in contrast to most of the literature, our results do not require weak mixing or exactness.

In direction (b), we show that certain axioms for quasicompactness described by Keller and Liverani [24] lift to the compact group extension setting, at the level of equivariant observations. We are claiming not that quasicompactness automatically lifts, but that certain sufficient axioms for quasicompactness lift. Since these axioms hold very generally, we are able to consider a large collection of examples. Thus we greatly generalise the applicability of the ideas in [16] while at the same time relaxing certain assumptions such as weak mixing.

REMARK 1.1. There are natural examples of dynamical systems for which our results apply although no power of the system is weak mixing. Suppose that $f: X \longrightarrow X$ is a mixing Axiom A diffeomorphism with invariant equilibrium measure $\mu$. Consider the $S^{1}$-extension $T: X \times S^{1} \longrightarrow X \times S^{1}$ given by $T(x, \theta)=$ $(f x, \theta+\alpha)$. If $\alpha$ is irrational, $T$ is ergodic with respect to $\mu \times \nu(\nu$ is Haar measure on $S^{1}$ ) but no power of $T$ is weak mixing. Let $\phi: X \times S^{1} \longrightarrow \mathbb{C}$ be an observation of the form $\phi(x, \theta)=e^{i m \theta} v(x)$ for some fixed integer $m$ and Hölder continuous $v: X \longrightarrow \mathbb{C}$.

It follows from the results in Section 5 that statistical limit laws such as the central limit theorem and weak invariance principle are valid. Furthermore, the almost sure invariance principle applies to this situation; see Remark 3.6(b).

The remainder of this paper is organised as follows. In Section 2, we discuss growth rates of ergodic sums at the level of isometries on a Hilbert space $H$. We show how a quasicompactness assumption leads directly to a martingale approximation, existence of the variance, and square root growth in $L^{2}$. (In this section, there is no dynamical system. The setting is analogous to the von Neumann mean ergodic theorem.)

In Section 3, we specialize to the case $H=L^{2}(X)$, where $f: X \longrightarrow X$ is an ergodic map satisfying a quasicompactness property. The central limit theorem (and much more) follows directly from the martingale approximation in Section 2.

In Section 4, we discuss the axiomatic framework of [24] guaranteeing quasicompactness of the Perron-Frobenius operator. Compact group extensions are introduced in Section 5 and it is shown that the axioms for quasicompactness in Section 4 lift to spaces of equivariant observations.

Finally, a number of applications are considered in Section 6.

\section{Quasicompact operators on Hilbert space}

Let $H$ be a Hilbert space and $U: H \longrightarrow H$ be an isometry. We are particularly interested in the case when $U$ is not invertible. Let $U^{*}: H \longrightarrow H$ be the adjoint of $U$ and note that $U^{*} U=I$. We recall the following basic properties of the spectra of $U$ and $U^{*}$.

(1) The eigenvalues of $U$ lie on the unit circle, and the spectra of $U$ and $U^{*}$ lie in the closed unit disk. 
(2) If $\alpha \in \mathbb{C}$ and $|\alpha|=1$, then $U v=\alpha v$ if and only if $U^{*} v=\bar{\alpha} v$. (One direction follows immediately from the fact that $U^{*} U=I$. In the other direction, one can compute directly that $\langle U v-\alpha v, U v-\alpha v\rangle=0$.)

(We note that in general $U^{*}$ may have eigenvalues $\alpha$ with $|\alpha|<1$.)

Given $v \in H$, we define $v_{N}=\sum_{j=1}^{N} U^{j} v$. By the mean ergodic theorem, $v_{N}=N \pi v+o(N)$ as $N \rightarrow \infty$, where $\pi: H \longrightarrow \operatorname{ker}(U-I)$ is the orthogonal projection. (That is, $\lim _{N \rightarrow \infty}(1 / N)\left\|v_{N}-N \pi v\right\|_{H}=0$.)

In this section, we are interested in obtaining more precise information on the growth of $v_{N}-N \pi v$ under a certain 'quasicompactness' hypothesis.

Definition 2.1. Let $F \subset H$ be a Banach space such that $U$ and $U^{*}$ restrict to bounded operators on $F$. The operator $U^{*}: F \longrightarrow F$ is quasicompact if $U^{*}: F \longrightarrow F$ has essential spectral radius $\rho<1$.

It follows from quasicompactness that $U^{*}$ has at most finitely many eigenvalues on the unit circle, that these eigenvalues have finite multiplicity, and that the rest of the spectrum is contained in a disk around the origin of radius less than 1 . (We note that our definition is slightly nonstandard, since we do not require that there exist eigenvalues $\alpha$ with $\rho<|\alpha| \leqslant 1$.)

By quasicompactness, there is a closed $U^{*}$-invariant splitting $F=F_{1} \oplus F_{2}$, where $F_{1}=\operatorname{ker}(U-I)=\operatorname{ker}\left(U^{*}-I\right)$. Again it is the case that $v_{N}=N v$ for all $v \in F_{1}$ and $\left\|v_{N}\right\|_{H}=o(N)$ for all $v \in F_{2}$.

TheOREM 2.2. Regarding $U$ as an operator on $F$ and $U^{*}$ as an operator on $F_{2}$, we have $F_{2}=\operatorname{ker} U^{*} \oplus \Im(U-I)$.

Proof. First, we show that $\operatorname{ker} U^{*} \cap \Im(U-I)=\{0\}$. Suppose that $U^{*} v=0$ and $v=U y-y$ where $y \in F$. Then $0=U^{*} v=y-U^{*} y$. It follows that $U y=y$ (since $U^{*} y=y$ implies that $\left.U y=y\right)$ and hence $v=0$ as required.

Second, we show that $F_{2}=\operatorname{ker} U^{*}+\Im(U-I)$. Let $F_{\alpha} \subset F_{2}$ be the sum of the eigenspaces corresponding to eigenvalues on the unit circle (other than 1 ). Note that $F_{\alpha}$ is finite-dimensional (possibly trivial). The quasicompactness hypothesis guarantees that we have the further closed $U^{*}$-invariant splitting $F_{2}=F_{\alpha} \oplus F_{3}$, where $U^{*}: F_{3} \longrightarrow F_{3}$ has spectral radius $\rho<1$.

If $v \in F_{\alpha}$ is an eigenfunction of $U$, so that $U v=\alpha v$, then $v=(\alpha-1)^{-1}(U-I) v$. Hence $F_{\alpha} \subset \Im(U-I)$.

It remains to show that $F_{3}=\operatorname{ker} U^{*}+\Im(U-I)$. Given $v \in F_{3}$, define $y=\sum_{j \geqslant 1}\left(U^{*}\right)^{j} v$. It follows from the spectral radius assumption that the series converges to $y \in F_{3}$. Now one can compute that $U^{*}\{v-U y+y\}=0$.

For reasons that will become clear in Section 3, we say that $w \in F$ is a martingale if $U^{*} w=0$. By Theorem 2.2, if $v \in F_{2}$, then there is a martingale approximation

$$
v=w+U y-y, \quad \text { where } w, y \in F_{2} \text { and } U^{*} w=0 .
$$

Since $v_{N}=w_{N}+U^{n} y-y$, many statistical properties for $v$ follow from the corresponding property for the martingale $w$. One result in this direction is the following. 
Corollary 2.3. Let $v \in F_{2}$ and write $v=w+U y-y$ as above. Define $\sigma=\|w\|_{H}$. Then $\left\|w_{N}\right\|_{H}=\sqrt{N} \sigma$ and

$$
\left\|v_{N}\right\|_{H}=\sqrt{N} \sigma+O(1) .
$$

In particular, $\sigma=\lim _{N \rightarrow \infty}(1 / \sqrt{N})\left\|v_{N}\right\|_{H}$.

Proof. Since $U^{*} w=0$, we compute that if $j>k$, then

$$
\left\langle U^{j} w, U^{k} w\right\rangle=\left\langle U^{j-k} w, w\right\rangle=\left\langle w,\left(U^{*}\right)^{j-k} w\right\rangle=0,
$$

and similarly for $j<k$. On the other hand, $\left\langle U^{j} w, U^{j} w\right\rangle=\langle w, w\rangle$. It follows that $\left\|w_{N}\right\|_{H}=\sqrt{N}\|w\|_{H}$. Next, consider the remainder term $r=U y-y$. Then $r_{N}=U^{N} y-y$ and so $\left\|r_{N}\right\|_{H} \leqslant 2\|y\|_{H}$.

REMARK 2.4. The requirement that $U$ restricts to an operator on $F$ is required only for Theorem 2.2. If we drop this requirement, then the decomposition (2.1) and Corollary 2.3 are still valid for all $v \in F_{2}$.

\section{Statistical properties of dynamical systems}

In this section, we apply the results in Section 2 to the case where $U$ is the Koopman operator associated to a measure-preserving transformation. Suppose that $X$ is a probability space with measure $m$ and $\sigma$-algebra $\mathcal{B}$. Let $f: X \longrightarrow X$ be a (noninvertible) measure-preserving transformation. We take $H=L^{2}(X)$ and define the isometry $U: H \longrightarrow H$ by $U v=v \circ f$. The adjoint $U^{*}$ satisfies $U^{*} U=I$ as before. In addition, $U U^{*} v=E\left(v \mid f^{-1} \mathcal{B}\right)$, where $E\left(\cdot \mid f^{-1} \mathcal{B}\right)$ is the conditional expectation operator.

Again, if $v \in H$, then we define $v_{N}=\sum_{j=1}^{N} U^{j} v=\sum_{j=1}^{N} v \circ f^{j}$. By the mean ergodic theorem, $v_{N}=N \pi v+o(N)$ in $H$, where $\pi: H \longrightarrow H$ is the orthogonal projection onto $\operatorname{ker}(U-I)$. (If $f: X \longrightarrow X$ is ergodic, then $\pi v=\int_{X} v d m$.)

As in Definition 2.1 and Remark 2.4, we assume quasicompactness, so there is a Banach space $F \subset H$ such that $U^{*}$ restricts to a bounded operator on $F$ with essential spectral radius less than 1. By Corollary 2.3, for all $v \in F$, we can define the variance $\sigma^{2}=\lim _{N \rightarrow \infty}(1 / N)\left\|v_{N}-N \pi v\right\|_{H}^{2}$.

TheOREM 3.1 (central limit theorem). Assume quasicompactness and assume that $f: X \longrightarrow X$ is ergodic. Let $v \in F$ with $\int_{X} v d m=0$. Then $(1 / \sqrt{N}) v_{N}$ converges in distribution to a normal distribution with mean zero and variance $\sigma^{2}$. That is,

$$
m\left\{x \in X: \frac{1}{\sqrt{N}} v_{N}<b\right\} \rightarrow \frac{1}{\sqrt{2 \pi} \sigma} \int_{-\infty}^{b} e^{-y^{2} / 2 \sigma^{2}} d y
$$

as $N \rightarrow \infty$ for all $b \in \mathbb{R}$.

Proof. Using the decomposition (2.1), we can write $v_{N}=w_{N}+U^{N} y-y$ almost everywhere, where $w, y \in F$ and $U^{*} w=0$. Moreover, since $y \in L^{2}$, it follows from the pointwise ergodic theorem that $U^{N} y=y \circ f^{N}=o\left(N^{1 / 2}\right)$ almost everywhere. Hence it suffices to prove that $(1 / \sqrt{N}) w_{N}$ converges in distribution to a normal distribution with mean zero and variance $\sigma^{2}$.

However, $U^{*} w=0$ implies that $E\left(w \mid f^{-1} \mathcal{B}\right)=0$. Passing to the natural extension $[\mathbf{3 2}]$, we obtain a bi-infinite ergodic stationary martingale $\left\{Y_{j}: j \in \mathbb{Z}\right\}$, 
where $Y_{-i}=w \circ f^{i}$ for $i \geqslant 0$ (cf. [16, Remark 3.12]). It follows from [5] that $(1 / \sqrt{|N|}) \sum_{j=0}^{N-1} Y_{j}$ converges to a normal distribution with mean zero and variance $\int Y_{1}^{2}$ as $N \rightarrow \pm \infty$. In particular, $(1 / \sqrt{N}) \sum_{j=1}^{N} w \circ f^{j}$ satisfies the central limit theorem with mean zero and variance $\sigma^{2}=\|w\|_{H}^{2}$.

We have the following criteria for degeneracy in the central limit theorem $\left(\sigma^{2}=0\right)$.

Proposition 3.2. Suppose that $v \in F$ and $\pi v=0$. Then the following hold.

(a) $\sigma^{2}=0$ if and only if $v=y \circ f-y$ for some $y \in F$.

(b) If $F \subset L^{\infty}$, then $\sigma^{2}=0$ if and only if $v_{N}=O(1)$ uniformly almost everywhere. Indeed, $\left|v_{N}\right|_{\infty} \leqslant 2|y|_{\infty}$.

(c) If $f: X \longrightarrow X$ is ergodic, then $\sigma^{2}=0$ if and only if $v_{N}=o(\sqrt{N})$ almost everywhere.

(d) If $f: X \longrightarrow X$ is ergodic, and $F \subset L^{p}$ for some $p>2$, then $\sigma^{2}=0$ if and only if $v_{N}=o\left(N^{1 / p}\right)$ almost everywhere.

Proof. Recall the decomposition $v=w+y \circ f-y$, where $U^{*} w=0$. By Corollary 2.3, $\sigma^{2}=0$ if and only if $w=0$ almost everywhere, proving part (a).

By part (a), if $\sigma^{2}=0$, then $v_{N}=y \circ f^{N}-y$. Part (b) follows immediately. As in the proof of Theorem 3.1, the pointwise ergodic theorem guarantees that $y \circ f^{N}=o\left(N^{1 / 2}\right)$, proving one direction of part (c). To prove the reverse direction, we note that if $v_{N}=o(\sqrt{N})$, then $(1 / \sqrt{N}) v_{N}$ converges to zero almost everywhere, and hence in distribution. However, since $f$ is ergodic, Theorem 3.1 guarantees convergence in distribution to a normal distribution with variance $\sigma^{2}$. Hence this is the degenerate normal distribution with $\sigma^{2}=0$. Part $(\mathrm{d})$ is proved in the same way as part (c).

REMARK 3.3. It is well known that the central limit theorem is degenerate if and only if $v=y \circ f-y$ for some $y \in L^{2}$. Our conditions in Proposition 3.2, which follow [16], are a substantial improvement. For example, suppose that $X$ is a topological space with open sets having positive measure, and that $f: X \longrightarrow X$, $v: X \longrightarrow \mathbb{R}$ are continuous. Under the assumptions of quasicompactness, ergodicity of $f: X \longrightarrow X$, and $F \subset L^{\infty}$, we have $\sigma^{2}=0$ if and only if there is a constant $C$ such that $\left|v_{N}(x)\right| \leqslant C$ for all $x \in X$ and $N \geqslant 1$. In particular, if $x$ is a periodic point of period $p$, then $v_{p}(x)=0$.

Set $W_{N}(0)=0$ and $W_{N}(t)=(1 / \sqrt{N}) v_{N t}=(1 / \sqrt{N}) \sum_{j=0}^{N t-1} v \circ f^{j}, t=1 / N, 2 / N, \ldots$. Linearly interpolating on each interval $[(r-1) / N, r / N], r \geqslant 1$, we obtain a sequence of random elements $W_{N} \in C([0, \infty), \mathbb{R})$. We have the weak invariance principle (which is a refinement of the central limit theorem):

THEOREM 3.4 (weak invariance principle). The sequence $\left\{W_{N}\right\}$ converges weakly in $C([0, \infty), \mathbb{R})$ to a Brownian motion with variance $\sigma^{2}$.

Proof. Billingsley $[6]$ proved the weak invariance principle for stationary ergodic $L^{2}$ martingales, so the result follows along the lines of Theorem 3.1.

TheOREM 3.5 (upper law of the iterated logarithm). Assume quasicompactness and assume that $f: X \longrightarrow X$ is ergodic. Suppose further that $F \subset L^{\infty}$. Let $v \in F$ 
with $\int_{X} v d m=0$. Then

almost surely.

$$
\limsup _{N \rightarrow \infty} \frac{v_{N}}{\sqrt{2 N \log \log N}} \leqslant \sigma
$$

Proof. Again, we write $v_{N}=w_{N}+U^{n} y-y$, where $U^{*} w=0$, and it suffices to prove the upper law of the iterated logarithm for the sequence $\left\{w_{N}\right\}$. As pointed out in $[\mathbf{1 6}$, Section $3(\mathrm{c})]$, the condition $U^{*} w=0$ implies that $\left\{w_{N}\right\}$ is a "weakly multiplicative sequence'. Since $w \in F \subset L^{\infty}$, the result follows by [34].

REMARK 3.6. (a) The full law of the iterated logarithm is the similar conclusion with $\leqslant$ replaced by $=$. We do not know whether the law of the iterated logarithm holds under our hypotheses, or whether it is possible to remove the $L^{\infty}$ assumption.

(b) Passing to the natural extension [32], it follows from the methods in [16] that the law of the iterated logarithm (and much more, including the almost sure invariance principle) can be proved in backwards time. Moreover, the $L^{\infty}$ assumption is not required.

In certain situations, such as for ergodic compact Lie group extensions of Axiom A base dynamics, these statistical properties can be deduced a fortiori in the correct time direction [16]. The approach in [16] requires two ingredients, that (i) there is a method for passing from invertible transformations to noninvertible transformations without losing too much regularity in the observations, and (ii) the class of dynamical systems is closed under time reversal. For the example in Remark 1.1, the almost sure invariance principle can be obtained in this way.

(c) The difficulty with time directions described above is a possibly serious limitation of the martingale approximation approach to proving the almost sure invariance principle [16]. The same issue arises in Conze and Le Borgne [14]. An alternative approach to proving the almost sure invariance principle is presented in Hofbauer and Keller [22].

REMARK 3.7. Assume quasicompactness and assume in addition that $f: X \longrightarrow X$ is weak mixing. Then, by standard arguments, exponential decay of correlations holds for observations in $F$. Indeed, there are constants $C>0$ and $\rho \in(0,1)$ such that $\left\|\left(U^{*}\right)^{n} v-\int v\right\| \leqslant C \rho^{n}\|v\|$, for all $v \in F$ and $n \geqslant 1$. Hence

$$
\left|\int_{X} v \cdot w \circ f^{n} d m-\int_{X} v d m \int_{X} w d m\right| \leqslant C \rho^{n}\|v\||w|_{2},
$$

for all $v \in F, w \in L^{2}, n \geqslant 1$. It follows easily that if $v \in F$ and $\int_{X} v d m=0$, then the variance is given by $\sigma^{2}=\int_{X} v^{2} d m+2 \sum_{j=1}^{\infty} \int_{X} v \cdot v \circ f^{j} d m$.

\subsection{Vector-valued observations}

We now generalise to the case of vector-valued observations $v: X \longrightarrow \mathbb{R}^{d}$. We continue to consider a measure-preserving transformation $f: X \longrightarrow X$ with $H=$ $L^{2}(X)=L^{2}(X, \mathbb{R})$, and assume that $F \subset H$ is a Banach space such that $U^{*}: F \longrightarrow F$ is quasicompact. Define $H^{d}=L^{2}\left(X, \mathbb{R}^{d}\right)$ and $F^{d}=\left\{v=\left(v_{1}, \ldots, v_{d}\right)\right.$ : $\left.X \longrightarrow \mathbb{R}^{d} \mid v_{j} \in F, j=1, \ldots, d\right\}$.

The operator $U v=v \circ f$ acts on vector-valued observations and defines an isometry on $H^{d}$ and a linear operator on $F^{d}$. Similarly, $U^{*}$ acts component-wise on $H^{d}$ and $F^{d}$. It is immediate that $U^{*}: F^{d} \longrightarrow F^{d}$ has essential spectral radius $\rho<1$ 
so that quasicompactness holds with $F^{d} \subset H^{d}$. Hence the results of Section 2 apply to functions $v \in F^{d}$.

In particular, the scalar variance $\sigma^{2}=\lim _{N \rightarrow \infty}(1 / N)\left\|v_{N}\right\|_{H^{d}}^{2}$ is defined for all $v \in F_{2}^{d}$. However, it is natural (following Field, Melbourne and Török [16]) to define the $d \times d$ covariance matrix

$$
\Sigma=\lim _{N \rightarrow \infty} \frac{1}{N} \int_{X} v_{N} \cdot v_{N}^{\mathrm{T}} d m \quad \text { (outer product). }
$$

Note that this limit is well defined when $U^{*} v=0$, in which case $\Sigma=$ $\int_{X} v \cdot v^{\mathrm{T}}=(1 / N) \int_{X} v_{N} \cdot v_{N}^{\mathrm{T}}$ for all $N$. Hence $\Sigma$ is well defined for all $v \in F_{2}^{d}$. Moreover, $\Sigma_{j k}=E\left(Y_{j} Y_{k}\right)$, and hence $\Sigma$ is symmetric and $\langle\Sigma x, x\rangle \geqslant 0$ for all $x \in \mathbb{R}^{d}$.

Next, we suppose further that $f: X \longrightarrow X$ is ergodic. Let $v \in F_{2}^{d}$ so that $\int_{X} v d m=0$. Given $c \in \mathbb{R}^{d}$, we have the decomposition $c \cdot v=c \cdot w+o\left(N^{1 / 2}\right)$ almost everywhere, where $U^{*}(c \cdot w)=0$. As in Theorem $3.1,(1 / \sqrt{N}) c \cdot v_{N}$ converges in distribution to a normal distribution with mean zero and variance $\sigma_{c}^{2}=|c \cdot w|_{2}^{2}=$ $c^{\mathrm{T}} \Sigma c$. By the Cramer-Wold technique (see for example [7, Theorem 29.4]), this implies that $(1 / \sqrt{N}) v_{N}$ converges in distribution to a $d$-dimensional normal distribution with mean zero and covariance matrix $\Sigma$. The distribution is nondegenerate if $\Sigma$ is nonsingular.

Similarly, the $d$-dimensional version of the weak invariance principle is valid for all $v \in F^{d}$ with $\int_{X} v d m=0$ when $f: X \longrightarrow X$ is ergodic. In addition, if $F \subset L^{\infty}$, then the upper law of the iterated logarithm holds for $c \cdot v$ for all $c \in \mathbb{R}^{d}$. If $f: X \longrightarrow X$ is weak mixing, then we obtain a $d$-dimensional analogue of exponential decay of correlations $[\mathbf{1 6}]$.

\section{Quasicompactness and the Perron-Frobenius operator}

In this section, we interpret the operator $U^{*}: L^{2} \longrightarrow L^{2}$ as the restriction of the Perron-Frobenius operator $P: L^{1} \longrightarrow L^{1}$ associated to the measure-preserving transformation $f: X \longrightarrow X$. Following Keller and Liverani [24], we give an axiomatic approach to quasicompactness.

As before, we let $X$ be a probability space with measure $m$ and $\sigma$-algebra $\mathcal{B}$, and $f: X \longrightarrow X$ is a (noninvertible) measure-preserving transformation. Given $1 \leqslant p \leqslant \infty$, we define the Koopman operator $U: L^{p} \longrightarrow L^{p}$ by $U v=v \circ f$. For each $p$, $U: L^{p} \longrightarrow L^{p}$ is an isometry.

Given $v \in L^{1}$, we define $P v$ by demanding that

$$
\int_{X} P v \cdot w d m=\int_{X} v \cdot U w d m=\int_{X} v \cdot w \circ f d m
$$

for all $w \in L^{\infty}$. The operator $P: L^{1} \longrightarrow L^{1}$ is called the Perron-Frobenius operator. Clearly, this also defines $P: L^{p} \longrightarrow L^{p}$ for all $1 \leqslant p \leqslant \infty$ (restricting to $w \in L^{q}$ where $1 / p+1 / q=1)$. When $p \geqslant 2$, the operator $P$ coincides with the operator $U^{*}$ in Section 2.

We assume the following.

(F1) $F$ and $F^{\prime}$ are Banach spaces, where $F \subset F^{\prime} \subset L^{1}$ with norms \|\|$\geqslant \mid$ respectively, and $F$ is densely embedded in $F^{\prime}$.

(F2) $F \subset L^{2}$ and \|\|$\geqslant||_{2}$.

(F3) $\{v \in F:\|v\|=1\}$ is compact in $F^{\prime}$. 
(F4) The Perron-Frobenius operator $P$ restricts to a bounded operator on $F$ and $F^{\prime}$. Moreover, there is a constant $C_{0}>0$ such that $\left|P^{n}\right| \leqslant C_{0}$ for all $n \geqslant 1$.

(F5) Lasota-Yorke inequality: for some $n_{0} \geqslant 1$, there are constants $D_{0}>0$ and $\theta_{0} \in(0,1)$ such that $\left\|P^{n_{0}} v\right\| \leqslant D_{0}|v|+\theta_{0}\|v\|$ for all $v \in F$.

REMARK 4.1. If $F^{\prime}=L^{p}$ for some $1 \leqslant p \leqslant \infty$ or $F^{\prime}=C(X)$, then hypothesis (F4) is automatically satisfied with $C_{0}=1$.

EXAMPLE 4.2. In Section 6, we consider a number of examples where (F1)-(F5) are satisfied. One such example is that of Hölder observations on a one-sided subshift of finite type. For details, see Ruelle [33], Bowen [8] or Parry and Pollicott [31]. Let $\sigma: X \longrightarrow X$ denote an irreducible (not necessarily aperiodic) subshift of finite type. Here $X \subset\{1, \ldots, k\}^{\mathbb{N}}$ for some $k$. Fix $\theta \in(0,1)$ and define $d_{\theta}(x, y)=\theta^{N}$, where $N \geqslant 1$ is least such that $x_{i}=y_{i}$ for $i<N$. Let $F_{\theta}$ be the space of functions that are Lipschitz with respect to this metric. Let $|g|_{\theta}$ denote the Lipschitz constant for $g \in F_{\theta}$ and define the norm $\|g\|_{\theta}=|g|_{\infty}+|g|_{\theta}$. Then $F_{\theta}$ is a Banach space. Moreover, taking $F=F_{\theta}$ and $F^{\prime}=C(X)$, it is immediate that (F1) and (F2) are valid, while (F3) follows from the Arzela-Ascoli theorem. By Remark 4.1, (F4) is automatic with $C_{0}=1$. Finally, the 'basic inequality' [31, Proposition 2.1] guarantees that (F5) holds for a large class of measures. (Technically speaking, (F5) holds whenever $m$ is an equilibrium measure corresponding to a potential $g \in F$. The Perron-Frobenius operator is the Ruelle transfer operator corresponding to a normalized version of g.)

We note that $F_{\theta}$ is a Banach algebra. Indeed, if $f, g \in F_{\theta}$, then $|f g|_{\theta} \leqslant|f|_{\infty}\|g\|_{\theta}+$ $|g|_{\infty}\|f\|_{\theta}$. Since $|f g|_{\infty} \leqslant|f|_{\infty}|g|_{\infty}$, it follows that $\|f g\|_{\theta} \leqslant\|f\|_{\theta}\|g\|_{\theta}$.

EXAMPLE 4.3. Another example is that of piecewise expanding maps of an interval $[\mathbf{1 0}, \mathbf{2 5}]$. Recall that a function $g:[0,1] \longrightarrow \mathbb{R}$ is of bounded variation, $g \in B V$, if

$$
\operatorname{var}(g)=\sup _{0 \leqslant t_{0}<t_{1}<\ldots<t_{k} \leqslant 1} \sum_{j=1, \ldots, k}\left|g\left(t_{j}\right)-g\left(t_{j-1}\right)\right|<\infty .
$$

The norm $\|g\|_{B V}=|g|_{1}+\operatorname{var}(g)$ is equivalent to $|g|_{\infty}+\operatorname{var}(g)$ (since $|g|_{1} \leqslant|g|_{\infty} \leqslant$ $\left.|g|_{1}+\operatorname{var}(g)\right)$ and $B V$ is a Banach space. If $f, g \in B V$, then $\operatorname{var}(f g) \leqslant|f|_{\infty} \operatorname{var}(g)+$ $|g|_{\infty} \operatorname{var}(f)$. A straightforward calculation shows that $\|f g\|_{B V} \leqslant 2\|f\|_{B V}\|g\|_{B V}$. Hence $B V$ is a Banach algebra. Let $F=B V$ and $F^{\prime}=L^{1}$.

Again, (F1), (F2) and (F4) are immediate, while (F3) is standard (see for example [22, Lemma 5]). Condition (F5) is discussed in detail in Section 6.

Proposition 4.4. Suppose that (F4) is valid. Then (F5) is equivalent to the condition that there are constants $E>0, \theta \in(0,1)$ such that $\left\|P^{n} v\right\| \leqslant E(|v|+$ $\left.\theta^{n}\|v\|\right)$, for all $v \in F, n \geqslant 1$.

Proof. If the conclusion holds, then choose $n_{0}$ large so that $\theta_{0}=\theta^{n_{0}} E<1$. Conversely, suppose that (F4) and (F5) hold. By induction,

$$
\left\|P^{j n_{0}} v\right\| \leqslant C_{0} D_{0}\left(1+\theta_{0}+\ldots+\theta_{0}^{j}\right)|v|+\theta_{0}^{j}\|v\| \leqslant C^{\prime}|v|+\theta^{j n_{0}}\|v\|
$$


where $C^{\prime}=C_{0} D_{0} /\left(1-\theta_{0}\right)$ and $\theta=\theta_{0}^{1 / n_{0}}$. Write $n=j n_{0}+k$, where $k<n_{0}$. Then $\left\|P^{n} v\right\| \leqslant C^{\prime}\left|P^{k} v\right|+\theta^{-1} \theta^{n}\left\|P^{k} v\right\|$, so the result follows with $E \geqslant C^{\prime} C_{0}$ and $E \geqslant \theta_{0}^{-1} \max \left\{\|P\|,\left\|P^{2}\right\|, \ldots,\left\|P^{n_{0}}\right\|\right\}$.

It follows easily that $P$ has spectral radius at most 1 in $F$ and $F^{\prime}$.

Theorem 4.5. Assume (F1)-(F5). Then the essential spectral radius $\rho_{\mathrm{ess}}$ of $P: F \longrightarrow F$ is strictly less than 1 . In fact $\rho_{\mathrm{ess}} \leqslant \theta_{0}^{1 / n_{0}}$, where $\theta_{0}, n_{0}$ are as in (F5) and $\rho_{\text {ess }} \leqslant \theta$, where $\theta$ is as in Proposition 4.4 .

Proof. See Hennion [18]. (See also $[\mathbf{1 9}, \mathbf{2 4}]$.)

Thanks to Theorem 4.5, we can apply the results of Section 2 (with $H=L^{2}, F$ as given, and $F^{\prime}$ disregarded from now on) to investigate the sequence of partial sums $v_{N}=\sum_{j=0}^{N-1} v \circ f^{j}$, where $v \in F$. Consider the $P$-invariant splitting $F=F_{1} \oplus F_{2}$, where $F_{1}=\operatorname{ker}(U-I)=\operatorname{ker}(P-I)\left(\right.$ recall $\left.P=U^{*}\right)$. Since $v_{N}=N v$ when $v \in F_{1}$, we restrict attention to $v \in F_{2}$. Then Corollary 2.3 and Remark 2.4 are valid. In particular, the variance $\sigma^{2}=\lim _{N \rightarrow \infty}(1 / N)\left|v_{N}\right|_{2}^{2}$ is defined for all $v \in F_{2}$, and $\left|v_{N}\right|_{2}=\sqrt{N} \sigma+O(1)$.

If we assume further that $f: X \longrightarrow X$ is ergodic, then the conclusions of Section 3 hold.

Theorem 4.6. Assume (F1)-(F5) and assume that $f: X \longrightarrow X$ is ergodic. Suppose that $v \in F$ has mean zero. Define $\sigma^{2}=\lim _{N \rightarrow \infty}(1 / N)\left|v_{N}\right|_{2}^{2}$. Then the following hold.

(a) $\left\{v_{N}\right\}$ satisfies the central limit theorem and weak invariance principle with variance $\sigma^{2}$.

(b) $\sigma^{2}=0$ if and only if $v$ is a coboundary in $F$, and if and only if $v_{N}=o\left(N^{1 / 2}\right)$ almost everywhere. If $F \subset L^{\infty}$, then $\sigma^{2}=0$ if and only if $v_{N}=O(1)$ uniformly almost everywhere.

(c) If $F \subset L^{\infty}$, then the upper law of the iterated logarithm holds for $\left\{v_{N}\right\}$.

(d) If $T$ is weak mixing, then we obtain exponential decay of correlations.

\subsection{Vector-valued observations}

In this section, we have deduced statistical properties of real-valued observations from quasicompactness of the operator $P: F \longrightarrow F$, where $F$ is a Banach space of real-valued observations. Statistical properties of vector-valued observations in $F^{d}$ can be proved just as in Section 3(c).

It is also immediate that hypotheses (F1)-(F5), which imply quasicompactness for $P: F \longrightarrow F$, also imply quasicompactness for $P: F^{d} \longrightarrow F^{d}$. (Clearly, the essential spectral radius on $F$ is less than 1 if and only if the essential spectral radius on $F^{d}$ is less than 1.$)$

Another way to see quasicompactness on $F^{d}$ is to note that hypotheses (F1)(F5) immediately extend to the Banach spaces $F^{d} \subset\left(F^{\prime}\right)^{d} \subset L^{1}\left(X, \mathbb{R}^{d}\right)$, so that Theorem 4.5 can be applied directly on $F^{d}$. The observation that (F1)-(F5) hold for $F^{d} \subset\left(F^{\prime}\right)^{d}$ is crucial in Section 5 . 


\subsection{Transfer operators}

So far, we have considered the Perron-Frobenius operator $P$ corresponding to an $f$-invariant measure $m$. In applications, quasicompactness is often proved first for a preliminary Perron-Frobenius operator $A$ corresponding to a 'reference measure' $\ell$ that is not $f$-invariant. See Section 6 for examples. We shall refer to $A$ as a transfer operator to distinguish it from the Perron-Frobenius operator $P$. The two measures are related by $d m=\varphi d \ell$ where the density function $\varphi>0$ is a fixed point for the transfer operator $(A \varphi=\varphi)$. Hence $P v=\varphi^{-1} A(\varphi v)$.

For many (but not all) applications, quasicompactness for $A$ immediately implies quasicompactness for $P$. If multiplication by $\varphi$ induces linear isomorphisms on $F^{\prime}$ and $F$, then we have the following useful result.

Lemma 4.7. Suppose that $A, B$ and $M$ are linear operators on both $F$ and $F^{\prime}$ such that $M: F^{\prime} \longrightarrow F^{\prime}$ and $M: F \longrightarrow F$ are linear isomorphisms and $B=M^{-1} A M$. Then $B: F^{\prime} \longrightarrow F^{\prime}$ satisfies (F4) and/or (F5) if and only if $A$ does.

Proof. First, suppose that $A$ satisfies (F4). Since $\left|A^{n}\right| \leqslant C_{0}$ for all $n,\left|B^{n}\right|=$ $\left|M^{-1} A^{n} M\right| \leqslant C_{0}\left|M^{-1}\right||M|$, so that $B$ satisfies (F4) with constant $C_{0}\left|M^{-1}\right||M|$. Next, we verify (F5). By Proposition 4.4, $\left\|A^{n} v\right\| \leqslant E\left(|v|+\theta^{n}\|v\|\right)$ and so

$$
\begin{aligned}
\left\|B^{n} v\right\| & =\left\|M^{-1} A^{n} M v\right\| \leqslant\left\|M^{-1}\right\|\left\|A^{n} M v\right\| \leqslant E\left\|M^{-1}\right\|\left(|M v|+\theta^{n}\|M v\|\right) \\
& \leqslant E\left\|M^{-1}\right\|\left(\left|M\left\|v \mid+\theta^{n}\right\| M\|\| v \|\right) \leqslant E\left\|M^{-1}\right\|(|M|+\|M\|)\left(|v|+\theta^{n}\|v\|\right),\right.
\end{aligned}
$$

so that $B$ satisfies the condition in Proposition 4.4 with constant $E\left\|M^{-1}\right\|(|M|+$ $\|M\|)$. Hence $B$ satisfies (F5).

\section{Compact group extensions}

In this section, we consider compact group extensions. The aim is to establish statistical properties of equivariant vector-valued observations which were introduced in [28] and occur naturally in applications to dynamical systems with symmetry.

Our treatment closely follows [16], which considered group extensions of Axiom A diffeomorphisms. In [16], use was made of the equivariant Ruelle operator which was studied by Parry and Pollicott in $[\mathbf{3 0}, \mathbf{3 1}]$. More generally, we consider an equivariant Perron-Frobenius operator which is a twisted version of the usual Perron-Frobenius operator. Our main results generalise results of $[\mathbf{1 6}]$ in the Axiom A setting, with the improvements that we require neither weak mixing nor that $G$ is connected.

Suppose that $f$ is a measure-preserving transformation on $(X, \mu)$. We assume that the Banach spaces $F \subset F^{\prime} \subset L^{1}(X, \mathbb{R})$ satisfy axioms (F1)-(F5) in Section 4. These properties are inherited by $F^{d} \subset\left(F^{\prime}\right)^{d} \subset L^{1}\left(X, \mathbb{R}^{d}\right)$. From now on, we write $F$ instead of $F^{d}$ and $F^{\prime}$ instead of $\left(F^{\prime}\right)^{d}$, so that $F \subset F^{\prime} \subset L^{1}\left(X, \mathbb{R}^{d}\right)$.

Let $G$ be a compact Lie group with Haar measure $\nu$. Given $h: X \longrightarrow G$ measurable, consider the skew product $T: X \times G \longrightarrow X \times G$ given by $T(x, g)=(f x, g h(x))$. Then $T$ is a measure-preserving transformation on $(X \times G, m)$, where $m=\mu \times \nu$.

Suppose that $G$ acts orthogonally on $\mathbb{R}^{d}$. For each $g \in G$, write $M_{g} v=g v$, and given $h: X \longrightarrow G$, write $\left(M_{h} v\right)(x)=h(x) v(x)$. Note that $M_{h}$ is an isometry on $L^{p}\left(X, \mathbb{R}^{d}\right)$ for all $p \geqslant 1$. Let $h_{n}(x)=h(x) h(f x) \ldots h\left(f^{n-1} x\right)$. To obtain control over 
the norms | | and \|\| on $F^{\prime}$ and $F$ under the action of $M_{h_{n}}$, we restrict to measurable cocycles $h: X \longrightarrow G$ that satisfy the following.

(G1) For all $n \geqslant 1, M_{h_{n}}^{-1}$ is a bounded operator on $F^{\prime}$. Moreover, there is a constant $C_{1}>0$ such that $\left|M_{h_{n}}^{-1}\right| \leqslant C_{1}$ for all $n \geqslant 1$.

(G2) Let $n_{0}$ and $\theta_{0}$ be as in (F5). Then $M_{*}=M_{h_{n_{0}}}^{-1}$ is a bounded operator on $F$, and moreover there exist constants $D_{1}>0$ and $c \in\left(0,1 / \theta_{0}\right)$ such that $\left\|M_{*} v\right\| \leqslant D_{1}|v|+c\|v\|$ for all $v \in F$.

REMARK 5.1. Suppose that $f: X \longrightarrow X$ and $h: X \longrightarrow G$ are measurable. Since $G$ is compact, $h \in L^{\infty}(X, G)$ automatically with $|h|_{\infty}=1$. If $F^{\prime}=L^{p}\left(X, \mathbb{R}^{d}\right)$ for some $1 \leqslant p \leqslant \infty$, then (G1) is satisfied with $C_{1}=1$. The same is true if $f$ and $h$ are continuous and $F^{\prime}=C\left(X, \mathbb{R}^{d}\right)$. Hence $(\mathrm{G} 1)$ is satisfied in Examples 4.2 and 4.3.

ExAmple 5.2. Continuing Example 4.2, if $F=F_{\theta}\left(X, \mathbb{R}^{d}\right)$ and $F^{\prime}=L^{\infty}\left(X, \mathbb{R}^{d}\right)$, then it is natural to restrict to cocycles $h: X \longrightarrow G$ that are Lipschitz with respect to the metric $d_{\theta}$ on $X$. We denote the space of such cocycles by $F_{\theta}(X, G)$. Then $\left|M_{*} v\right|_{\theta} \leqslant\left|M_{*}\right|_{\theta}|v|_{\infty}+\left|M_{*}\right|_{\infty}|v|_{\theta}=\left|M_{*}\right|_{\theta}|v|_{\infty}+|v|_{\theta}$, and so $\left\|M_{*} v\right\|_{\theta}=\left|M_{*} v\right|_{\infty}+$ $\left|M_{*} v\right|_{\theta} \leqslant\left\|h_{n_{0}}^{-1}\right\|_{\theta}|v|_{\infty}+\|v\|_{\theta}$. Hence (G2) is satisfied for $h \in F_{\theta}(X, G)$ with $D_{1}=\left\|h_{n_{0}}^{-1}\right\|_{\theta}$ and $c=1$.

Example 5.3. Continuing Example 4.3, if $F=B V$ (with $X=[0,1]$ ), then it is natural to restrict to BV cocycles $h: X \longrightarrow G$. In particular, for certain classes of piecewise monotone functions $f: X \longrightarrow X$, including functions whose domains of monotonicity form a finite partition of $[0,1]$, it is easily seen that $M_{h_{n}}^{-1}: B V \longrightarrow B V$ is a bounded operator for each $n$, and

$$
\left\|M_{h_{n}}^{-1}\right\|=\left\|h_{n}^{-1}\right\|_{B V}=1+\operatorname{var}\left(h_{n}^{-1}\right)
$$

In contrast to the previous example, it is necessary to add a further restriction on $h$. Let $n_{0}$ and $\theta_{0}$ be the constants in (F5). We assume that $\operatorname{var}\left(h_{n_{0}}^{-1}\right)<\left(1 / \theta_{0}\right)-$ 1. (Equivalently, $\left\|h_{n_{0}}^{-1}\right\|_{B V}=c<1 / \theta_{0}$.) Then $\left\|M_{*}\right\| \leqslant c<1 / \theta_{0}$ and (G2) is satisfied with $D_{1}=c=\left\|h_{n_{0}}^{-1}\right\|_{B V}$.

Alternatively, if $f: X \longrightarrow X$ is piecewise Lipschitz, then we can take $h: X \longrightarrow G$ to be piecewise Lipschitz with no restriction on the Lipschitz constant $L(h)$. The crucial estimate is $\operatorname{var}\left(M_{*} v\right) \leqslant L\left(h_{n_{0}}^{-1}\right)|v|_{1}+\operatorname{var}(v)$. (Note that the $L^{1}$ norm appears in this estimate, whereas before we had only the $L^{\infty}$ norm. The function spaces $F^{\prime}=L^{1}, F=B V$ are as before.)

For $h: X \longrightarrow G$ measurable, we define the equivariant Perron-Frobenius operator $P_{h}: L^{1}\left(X, \mathbb{R}^{d}\right) \longrightarrow L^{1}\left(X, \mathbb{R}^{d}\right)$ by $P_{h} v=P M_{h}^{-1} v$. Observe that $P_{h}^{n}=P^{n} M_{h_{n}}^{-1}$.

Proposition 5.4. Assume (F1), (F4), (F5), (G1) and (G2). Then the following hold.

(a) There is a constant $C_{1}^{\prime}>0$ such that $\left|P_{h}^{n}\right| \leqslant C_{1}^{\prime}$ for all $h \in H, n \geqslant 1$.

(b) There are constants $D_{1}^{\prime}>0$ and $\theta_{0}^{\prime} \in(0,1)$ such that $\left\|P_{h}^{n_{0}} v\right\| \leqslant D_{1}^{\prime}|v|+\theta_{0}^{\prime}\|v\|$ for all $v \in F$. 
Proof. For $v \in F^{\prime},\left|P_{h}^{n} v\right|=\left|P^{n} M_{h_{n}}^{-1} v\right| \leqslant C_{0}\left|M_{h_{n}}^{-1} v\right| \leqslant C_{0} C_{1}|v|$, proving (a). To prove (b), we compute that

$$
\left\|P_{h}^{n_{0}} v\right\|=\left\|P^{n_{0}} M_{\star} v\right\| \leqslant D_{0}\left|M_{\star} v\right|+\theta_{0}\left\|M_{\star} v\right\| \leqslant D_{0} C_{1}|v|+\theta_{0}\left(D_{1}|v|+c\|v\|\right),
$$

so the result follows with $D_{1}^{\prime}=D_{0} C_{1}+\theta_{0} D_{1}$ and $\theta_{0}^{\prime}=c \theta_{0}$.

Let $L_{G}^{p}$ consist of equivariant observations $\phi: X \times G \longrightarrow \mathbb{R}^{d}$ of the form $\phi(x, g)=M_{g} v(x)$, where $v \in L^{p}\left(X, \mathbb{R}^{d}\right)$, and define $|\phi|_{p}=|v|_{p}$. Symbolically, we can write $L_{G}^{p}=g \cdot L^{p}\left(X, \mathbb{R}^{d}\right)$.

Proposition 5.5. Let $\widehat{P}: L^{1}\left(X \times G, \mathbb{R}^{d}\right) \longrightarrow L^{1}\left(X \times G, \mathbb{R}^{d}\right)$ denote the Perron-Frobenius operator corresponding to the $G$-extension $T: X \times G \longrightarrow X \times G$. Then $\left.\widehat{P}\right|_{L_{G}^{1}}=M_{g} P_{h} M_{g}^{-1}$.

Proof. Let $\phi=g \cdot v \in L_{G}^{1}$ and $\psi=g \cdot w \in L_{G}^{\infty}$. Then

$$
\begin{aligned}
\int_{X \times G} M_{g} P_{h} M_{g}^{-1} \phi \cdot \psi^{\tau} d m & =\int_{X} P_{h} v \cdot w^{\tau} d \mu=\int_{X} P M_{h}^{-1} v \cdot w^{\tau} d \mu \\
& =\int_{X} M_{h}^{-1} v \cdot w^{\tau} \circ f d \mu=\int_{X} v \cdot\left(M_{h} w \circ f\right)^{\tau} d \mu \\
& =\int_{X \times G} M_{g} v \cdot\left(M_{h} w \circ f\right)^{\tau} M_{g}^{\tau} d m=\int_{X \times G} \phi \cdot \psi^{\tau} \circ T d m .
\end{aligned}
$$

Similarly, define the spaces $F_{G}=g \cdot F$ with norm $\|g \cdot v\|=\|v\|$ and so on.

Proposition 5.6. The operator $\widehat{P}=M_{g} P_{h} M_{g}^{-1}$ restricts to $F_{G}^{\prime}$ and $F_{G}$ and satisfies properties (F1)-(F5).

Proof. Properties (F1)-(F3) are immediate since $F_{G}^{\prime}$ and $F_{G}$ are isomorphic to $F^{\prime}$ and $F$. Properties (F4) and (F5) are Proposition 5.4(a) and (b), respectively.

We can now apply Theorem 4.5 to deduce that $\widehat{P}: F_{G} \longrightarrow F_{G}$ is quasicompact. We obtain the following conclusions.

Theorem 5.7. Assume (F1)-(F5) for $f: X \longrightarrow X$ and (G1),(G2) for $h$ : $X \longrightarrow G$. Suppose that $T: X \times G \longrightarrow X \times G$ is ergodic and that $\phi \in F_{G}$ has mean zero. Then the following hold.

(a) The $d \times d$ covariance matrix $\Sigma=\lim _{N \rightarrow \infty}(1 / N) \int_{X \times G} \phi_{N} \cdot \phi_{N}^{\mathrm{T}} d m$ is well defined and $\Sigma: \mathbb{R}^{d} \longrightarrow \mathbb{R}^{d}$ commutes with the action of $G$ on $\mathbb{R}^{d}$.

(b) $\left\{\phi_{N}\right\}$ satisfies $d$-dimensional versions of the central limit theorem and weak invariance principle on $X \times G$ with covariance matrix $\Sigma$.

(c) $\operatorname{det} \Sigma=0$ if and only if there is a $G$-invariant subspace $V \subset \mathbb{R}^{d}$ such that $\pi_{V} \phi$ is a coboundary in $F_{G}$, and if and only if $\pi_{V} \phi_{N}=o\left(N^{1 / 2}\right)$. If $F \subset L^{\infty}$, then $\sigma^{2}=0$ if and only if there is a $G$-invariant subspace $V$ such that $\pi_{V} \phi_{N}=O(1)$.

(d) If $F \subset L^{\infty}$, then the upper law of the iterated logarithm holds for each component of $\left\{\phi_{N}\right\}$.

(e) If $T$ is weak mixing, then we obtain exponential decay of correlations.

Proof. The definition of $\Sigma$ and parts (b), (d) and (e) are immediate from quasicompactness and Section 3. The statement about $\Sigma$ commuting with $G$ and part (c) are proved as in $[\mathbf{2 8}]$ (see also [16]). 
REMARK 5.8. By [27], we obtain the stronger results that the central limit theorem, weak invariance principle and upper law of the iterated logarithm hold also on $X \times\left\{g_{0}\right\}$ for each fixed $g_{0} \in G$. In the remainder of this paper, we will not mention this explicitly.

\section{Applications}

In this section we give examples of dynamical systems to which our results apply.

Let $f: X \longrightarrow X$ be a mapping with certain regularity properties. Consider a transfer operator $A$ acting on sufficiently regular functions $v$ as $(A v)(x)=$ $\sum_{f y=x} g(y) v(y)$, where $g$ is a positive bounded function (again with certain regularity properties).

Suppose that $\ell$ is a Borel probability measure satisfying $A^{*} \ell=\ell$ (that is, $\int A^{*} v d \ell=\int v d \ell$ for all $\left.v\right)$. Then $\int A v w d \ell=\int v w \circ f d \ell$. Thus $A$ is the PerronFrobenius operator corresponding to the measure $\ell$. We do not assume that $\ell$ is $f$-invariant.

Now suppose that $\varphi>0$ is a fixed point $(A \varphi=\varphi)$ for $A$ acting on a suitable function space. Then we define the $f$-invariant measure $d m=\varphi d \ell$ and the corresponding Perron-Frobenius operator $P v=\varphi^{-1} A(\varphi v)$.

In the rest of this section, we refer to $\ell$ as a reference measure and $m$ as an equilibrium measure. Note that in earlier sections, we chose to work directly with $P$ and $m$, bypassing $A$ and $\ell$.

\subsection{One-dimensional monotone maps}

Hofbauer and Keller [22] analysed a class of endomorphisms of a totally ordered, order complete set $X$ (usually $X$ is taken to be the unit interval [0,1]). Here $f: X \longrightarrow X$ is piecewise monotonic and order-continuous and the transfer operator $A$ acts on the space of bounded measurable functions by $A v(x)=\sum_{f y=x} g(y) v(y)$, where $g$ is a function of bounded variation on $[0,1]$ with $0<g(x) \leqslant d<1$. They showed the existence of a reference Borel probability measure $\ell$ on $X$ satisfying $A^{*} \ell=\ell$ in the sense that $\int A v d \ell=\int v d \ell$ for all bounded measurable $v: X \longrightarrow \mathbb{R}$. Under these assumptions, there exists a maximal absolutely continuous invariant measure $d m=\varphi d \ell$, where $\varphi$ is a density of bounded variation.

Examples of the systems considered in [22] include the following.

(i) Lasota-Yorke maps: piecewise monotonic $C^{2}$ transformations $f$ of the unit interval $[0,1]$ which satisfy $\left|f^{\prime}\right|>1$ : In this setting, $\ell$ is Lebesgue measure and $g(x)=1 /\left|f^{\prime}(x)\right|$. For earlier results on these maps see $[\mathbf{9}, \mathbf{2 5}, \mathbf{2 6}, \mathbf{3 6}, \mathbf{3 8}]$.

(ii) Piecewise monotonic transformations $f$ on $[0,1]$ with $h_{\text {top }}(f)>0[\mathbf{2 0}, \mathbf{2 1}]$ : Setting $g(x)=\exp \left(-h_{\text {top }}(f)\right)<1$, it can be shown that there exists $\ell$ such that $A^{*} \ell=\ell$, leading to an $f$-invariant measure $d m=\varphi d \ell$ of maximal entropy.

(iii) The $\beta$-transformation $f x=\beta x(\bmod 1)$ on $[0,1]$ : Walters $[37]$ constructed equilibrium measures corresponding to Lipschitz potentials $\phi:[0,1] \longrightarrow \mathbb{R}$. The class of allowable potentials is extended in [22], and, defining $g(x)=$ $\exp \left(\sum_{i=1}^{n-1} \phi\left(f^{i} x\right)\right) / \lambda^{n}$ for suitable choices of $\lambda>0$ and $n \geqslant 1$, it is shown that there exists a Borel probability measure $\ell$ such that $A^{*} \ell=\ell$. Again, this leads to an $f$-invariant equilibrium measure $d m=\varphi d \ell$ with potential function $\phi$.

For the class of transformations (i) and (iii) above, the density $\varphi$, which is of bounded variation, is bounded above and below on the support of $m$. That is, there 
exists $C \geqslant 1$ such that $0<1 / C<\varphi<C$. The same is true for class (ii) under the assumption that $l(f I) / l(I)$ is bounded over intervals $I \subset[0,1]$ (here $l$ is Lebesgue measure). This was proved in an unpublished preprint of Keller $[\mathbf{2 3}]$ in the context of Lasota-Yorke maps, but it is easily seen that the proof generalizes to class (iii) and to class (ii) if we also require $\sup _{I \subset[0,1]} l(f I) / l(I)$ to be bounded. We let class (ii') denote the subset of class (ii) maps for which $\sup _{I \subset[0,1]} l(f I) / l(I)$ is bounded.

LEMma 6.1. Let $f:[0,1] \longrightarrow[0,1]$ be a one-dimensional piecewise monotone map and let $g \in B V$ with transfer operator $A: B V \longrightarrow B V$ given by $A v(x)=$ $\sum_{f y=x} g(y) v(y)$. Suppose that $f$ and $g$ fall into one of the three classes (i), (ii) and (iii). Let $\ell$ and $m$ be the corresponding reference and equilibrium measures with density $\varphi>0$ in $B V$. Then $A$ satisfies hypotheses (F1)-(F5) with $F^{\prime}=L^{1}(m)$ and $F=B V$. In particular, $A: B V \longrightarrow B V$ is quasicompact.

Suppose further that $\varphi^{-1} \in B V$ (which is certainly the case for the classes (i), (ii'), (iii)). Define the Perron-Frobenius operator $P v=\varphi^{-1} A(\varphi v)$ corresponding to the invariant measure $m$. Then $P$ satisfies hypotheses (F1)-(F5) with $F^{\prime}=L^{1}(m)$ and $F=B V$. In particular, $P: B V \longrightarrow B V$ is quasicompact.

Proof. We largely follow Hofbauer and Keller [22]. Conditions (F1)-(F3) have already been discussed in Example 4.3. Since $\varphi, \varphi^{-1} \in F$, it follows that $v \longmapsto \varphi v$ is a linear isomorphism on $F^{\prime}$ and $F$. (Indeed, it is clear that $|\varphi v|_{1} \leqslant|\varphi|_{\infty}|v|_{1}$, and $\|\varphi v\|_{B V} \leqslant 2\|\varphi\|_{B V}\|v\|_{B V}$ was established in Example 4.3.) Hence, by Lemma 4.7, it suffices to verify (F4) and (F5) for either $A$ or $P$.

Condition (F4) is immediate for $P$ by Remark 4.1. (Alternatively, see [22, Lemma 6].) The crucial condition (F5) is proved for $A$ in [22, Lemma 7].

We are now in a position to apply the results in Section 2-4. Assume that the equilibrium measure $m$ in Lemma 6.1 is ergodic. Let $v \in B V$ with $\int_{X} v d m=0$.

(1) The variance $\sigma^{2}=\lim _{N \rightarrow \infty}(1 / N) \int_{X} v_{N}^{2} d m$ exists, and $\sigma^{2}=0$ if and only if $v=\chi \circ f-\chi$ for some $\chi \in B V$ (or equivalently, $v_{N}$ is uniformly bounded). In particular, if $x \in X$ is a periodic point of period $k, f$ and $v$ are continuous at $f^{j} x$ for $j=1, \ldots, k$, and $v_{k}(x) \neq 0$, then $\sigma^{2}>0$.

(2) The sequence of partial sums $v_{N}$ satisfies the central limit theorem, weak invariance principle and upper law of the iterated logarithm.

(3) If in addition $m$ is weak mixing, then we obtain exponential decay of correlations.

REMARK 6.2. Under the assumption of weak mixing, various statistical results were obtained in $[\mathbf{2 2}]$. We have extended the central limit theorem, weak invariance principle and upper law of the iterated logarithm to the case where $m$ is ergodic but not necessarily weak mixing. Also, we have obtained strong conditions for nondegeneracy that are not present in $[\mathbf{2 2}]$.

6.1.1. G-extensions of one-dimensional monotone maps. We continue to suppose that $X=[0,1]$ and that $f: X \longrightarrow X$ is piecewise monotone. Suppose that $m$ is an ergodic measure belonging to classes (i), (ii') or (iii) above.

Now let $G$ be a compact Lie group acting on $\mathbb{R}^{d}$. Let $h: X \longrightarrow G$ be a BV cocycle and form the compact group extension $T(x, g)=(f x, g h(x))$. We suppose further that $\operatorname{var}(h)$ is sufficiently small, in the sense of Example 5.3, guaranteeing that $h$ 
satisfies (G1) and (G2). In addition, we assume that $T: X \times G \longrightarrow X \times G$ is ergodic with respect to $m \times \nu$, where $\nu$ is Haar measure on $G$.

As in Section 5 , we define the space $B V_{G}$ of $G$-equivariant observations $\phi(x, g)=$ $g v(x)$, where $v: X \longrightarrow \mathbb{R}^{d}$ is $B V$. Suppose that $\phi \in B V_{G}$ and $\int_{X \times G} \phi d(m \times \nu)=0$.

By Theorem 5.7, we have the following results.

(1) The covariance matrix $\Sigma=\lim _{N \rightarrow \infty}(1 / N) \int_{X \times G} \phi_{N} \phi_{N}^{\mathrm{T}} d(m \times \nu)$ exists. Moreover, $\operatorname{det} \Sigma=0$ if and only if there is a component of $\phi_{N}$ that is uniformly bounded almost everywhere.

(2) The sequence of partial sums $\phi_{N}$ satisfies the $d$-dimensional central limit theorem and weak invariance principle.

(3) The components of $\phi$ satisfy the upper law of the iterated logarithm.

(4) If in addition $m \times \nu$ is weak mixing, then we obtain exponential decay of correlations.

REMARK 6.3. Results of [29] show that compact group extensions of LasotaYorke maps are weak mixing for a residual, prevalent subset of Hölder compact group extensions of Lasota-Yorke maps. If $G$ is semisimple, then compact group extensions of Lasota-Yorke maps are weak mixing for an open, dense and prevalent subset of extensions. Hence the hypotheses required for our probabilistic properties to hold are 'typically' valid for extensions of Lasota-Yorke maps.

\subsection{Nonuniformly hyperbolic diffeomorphisms}

In this section we follow very closely the original exposition of Young [39], referring also to Baladi $[\mathbf{1}, \S 4.3]$. The tower approach is applicable to the following piecewise $C^{1+\epsilon}$ diffeomorphisms $\mathcal{F}$ defined on a Riemannian manifold $M$ :

(i) Lozi maps and certain piecewise hyperbolic maps $[\mathbf{1 2}, \mathbf{3 9}]$;

(ii) a class of Hénon maps [3, 4];

(iii) Poincaré maps of billiards with convex scatterers [39] and certain other dispersing billiards $[\mathbf{1 3}]$;

(iv) some partially hyperbolic diffeomorphisms with a mostly contracting direction $[\mathbf{1 1}, \mathbf{1 5}]$.

The approach also applies to

(v) $C^{2}$ unimodal maps satisfying conditions (H1) and (H2) of Young [39, §9.1].

For these systems a Markov tower $(\bar{f}, \bar{\Delta})$ is constructed. (Since most of our discussion is on a 'quotiented' tower we use a bar to denote the unquotiented tower. This is not the same notation as in Young $[\mathbf{3 9}, \S 9.1]$.) The set $\bar{\Delta}$ is partitioned into countably many levels $\left\{\bar{\Delta}_{j}\right\}_{j=0}^{\infty}$. The base $\bar{\Delta}_{0}$ is further partitioned into countably many subsets $\left\{\bar{\Delta}_{0, j}\right\}$ by a return time function $R: \bar{\Delta}_{0} \longrightarrow \mathbb{N}$ such that $R \mid \bar{\Delta}_{0, j}=R_{j}$ is constant and $\bar{f}$ is $C^{1+\epsilon}$ on each $\bar{\Delta}_{0, j}$. The map $\bar{f}$ moves each set $\bar{\Delta}_{0, j}$ up the tower until the level $\bar{\Delta}_{R_{j}-1}$ is reached, and

$$
\bar{f}^{R_{j}} \quad \operatorname{maps} \bar{\Delta}_{0, j}
$$

bijectively onto $\bar{\Delta}_{0}$. The levels $\bar{\Delta}_{l}$ are further subdivided so that the partition $P=\left\{\bar{\Delta}_{l, j}\right\}$ of $\bar{\Delta}$ has the Markov property. A separation time $s(\cdot, \cdot)$ is defined for all pairs $x, y$ in the same $\bar{\Delta}_{l, j} ; s(x, y)$ is the largest $n \geqslant 0$ such that $\bar{f}^{n} x$ lies in the same element of $P$ as $\bar{f}^{n} y$. It is assumed [39, Condition P4] that there 
exists $0<\alpha<1$ such that $d\left(\mathcal{F}^{n} x, \mathcal{F}^{n} y\right) \leqslant C \alpha^{s(x, y)-n}$ for all $y \in \gamma^{u}(x)$ (here $\gamma^{u}$ is an unstable disk or manifold [39, Definition 1]).

A non-invertible tower $(f, \Delta)$ is derived by quotienting $(\bar{f}, \bar{\Delta})$ along stable manifolds (the quotiented tower is not necessary for the unimodal maps described in $(\mathrm{v}))$. Denote this projection by $\bar{\pi}: \bar{\Delta} \longrightarrow \Delta$, and write corresponding objects under this quotient map without bars. The map $f^{R_{j}}: \Delta_{0, j} \longrightarrow \Delta_{0}$ is uniformly expanding. A reference measure $\ell$, equivalent to Lebesgue, is constructed on $\Delta[\mathbf{3 9}, \S 3]$.

By studying the transfer operator $A$ with weight $g=1 / \operatorname{Jac}(f)$ acting on a suitable space of functions, Young obtains an absolutely continuous invariant measure $d m=\varphi d \ell$, with density $C^{-1} \leqslant \varphi \leqslant C$ bounded above and below.

The measure $m$ lifts to an invariant measure $\bar{m}$ on $\bar{\Delta}$ and thence to an invariant measure $\mu$ for $\mathcal{F}: M \longrightarrow M$. We assume, following [39], that there exists $\epsilon>0$ such that $\sum_{l=0}^{\infty} m\left(\Delta_{l}\right) e^{2 \epsilon l}<\infty$ (equivalently, $\sum_{l=0}^{\infty} \ell\left(\Delta_{l}\right) e^{2 \epsilon l}<\infty$ ). (The scaling factor $2 \epsilon l$ rather than $\epsilon l$ ensures that the Banach space $F$ (to be defined) satisfies $F \subset L_{m}^{2}$.)

The underlying observations $v: M \longrightarrow \mathbb{R}$ are assumed to be Hölder continuous with fixed exponent $\gamma \in(0,1)$. Take $0<\beta<1$ such that $\beta \geqslant \max \left\{\sqrt{\alpha}, \alpha^{\gamma}\right\}$. This implies that $d(x, y) \leqslant \beta^{s(x, y)}$. In fact, $\beta^{s(x, y)}$ defines a metric on $\Delta$, as $\beta^{s(x, y)}=0$ implies $x=y$ and the triangle inequality is immediate from the definition of $s(x, y)$.

For $v: \Delta \longrightarrow \mathbb{R}$ measurable, define

$$
\|v\|_{\infty}=\sup _{l, j} \sup _{\Delta_{l, j}}|v| e^{-l \epsilon}, \quad\|v\|_{\beta}=\sup _{l, j}|v|_{l, j, \beta}
$$

where

$$
|v|_{l, j, \beta}=\left(\sup _{x, y \in \Delta_{l, j}} \frac{|v(x)-v(y)|}{\beta^{s(x, y)}}\right) e^{-\epsilon l} .
$$

Define $\|v\|=\|v\|_{\infty}+\|v\|_{\beta}$ and (see $[\mathbf{1}, \S 3.4$, p. 203]) define $F$ to be the Banach space of functions $v: \Delta \longrightarrow \mathbb{R}$ with $\|v\|<\infty$. Let $F^{\prime}=L_{m}^{1}(\Delta)$. The transfer operator $A$ is well defined on $F$ and on $F^{\prime}$. (If $\mathcal{F}$ is globally $C^{1+\epsilon}$, then the Koopman operator $U$ also acts on $F$ so that stronger results hold; see Remark 2.4.) As sketched below, conditions (F1)-(F5) are valid. Since $\beta^{s(x, y)}$ defines a metric on $\Delta$, elements of $F$ are Lipschitz on each level $\Delta_{l}$.

The density $\varphi$ lies in $F$, as does $\varphi^{-1}$. Additionally, $\varphi$ and $\varphi^{-1}$ are uniformly bounded and uniformly Lipschitz [39, Lemma 2]. Hence $v \longmapsto \varphi v$ is a linear isomorphism on $F^{\prime}$ and $F$ so that Lemma 4.7 is applicable.

(F1) It suffices to work on each level $\Delta_{l}$ separately. Note that $F$ restricted to $\Delta_{l}$ is densely embedded in $F^{\prime}$ (similarly restricted) since the space of Lipschitz functions on a compact measure metric space $(X, \mu)$ is densely embedded in $L_{\mu}^{1}(X)$.

(F2) If $v \in F$, then $|v|_{\Delta_{l}} \leqslant\|v\|_{\infty} e^{\epsilon l}$ and thus $\sum_{l} e^{2 \epsilon l} m\left(\Delta_{l}\right)<\infty$ implies that $F \subset L_{m}^{2}$. A suitable scaling of the norm \|\| yields \|\|$\geqslant||_{2}$.

(F3) Suppose that $\left\{v_{n}\right\}$ is a sequence in $F$ with $\left\|v_{n}\right\| \leqslant 1$. Restricting to $\Delta_{l}$, we have $\left\|v_{n} \mid \Delta_{l}\right\| \leqslant e^{\epsilon \ell}$. By the Arzela-Ascoli theorem, there exists $w: \Delta_{l} \longrightarrow \mathbb{R}$ with $\|w\| \leqslant e^{\epsilon \ell}$ and a subsequence with $\left\|v_{n} \mid \Delta_{l}-w\right\|_{\infty} \rightarrow 0$. Altogether, we obtain a function $w: \Delta \longrightarrow \mathbb{R}$ with $w \in F$ and $\|w\| \leqslant 1$. By a standard diagonal argument there is a single subsequence such that $\left\|\left(v_{n}-w\right) \mid \Delta_{l}\right\|_{\infty} \rightarrow 0$ for all $l$ and hence $\left\|\left(v_{n}-w\right) \mid \Delta_{l}\right\|_{1} \rightarrow 0$ for each $l$. Since $\sum_{l=0}^{\infty} m\left(\Delta_{l}\right)<\infty$, it follows that $\left\|v_{n}-w\right\|_{1} \rightarrow 0$.

(F4) It is immediate that $P$ satisfies (F4) by Remark 4.1. (By Lemma 4.7, it is also the case that $A$ satisfies (F4).) 
(F5) This condition is verified for $A$ in [1, Lemma 3.7; 39, Lemma 3]. Applying Lemma 4.7, we have $P$ satisfies (F5).

We are now once again in a position to apply the results in Sections 2-4. Assume that $m$ is ergodic and that $v \in F$ with $\int_{\Delta} v d m=0$.

(1) The variance $\sigma^{2}=\lim _{N \rightarrow \infty}(1 / N) \int_{\Delta} v_{N}^{2} d m$ exists, and $\sigma^{2}=0$ if and only if $v=\chi \circ f-\chi$ for some $\chi \in F$ (in particular, $v_{N}$ is uniformly bounded on each level $\Delta_{\ell}$ ). If $x \in \Delta$ is a periodic point of period $k$, and $v_{k}(x) \neq 0$, then $\sigma^{2}>0$.

(2) $v_{N}$ satisfies the central limit theorem and the weak invariance principle.

(3) If in addition $m$ is weak mixing, then we obtain exponential decay of correlations.

REMARK 6.4. The central limit theorem and exponential decay of correlations were obtained in Young [39]. The strong nondegeneracy results stated here are new. The weak invariance principle is not stated in [39] but it follows in a standard way from the set-up there. As far as we know, the upper law of the iterated logarithm remains open. Note that the condition $F \subset L^{\infty}$ in Theorem 3.5 is violated.

6.2.1. G-extensions. Suppose that a quotiented tower $(f, \Delta, m)$ has been constructed as above. Let $G$ be a compact Lie group acting on $\mathbb{R}^{d}$. Let $h: \Delta \longrightarrow G$ be uniformly Lipschitz and let $T: \Delta \times G \longrightarrow \Delta \times G$ denote the corresponding $G$ extension.

Hypothesis (G1) is immediate by Remark 5.1. It is easily seen that (G2) is satisfied with $D_{1}=1+L\left(h_{n_{0}}^{-1}\right)$ and $c=1$. We define the space $F_{G}$ of $G$-equivariant observations by $\phi(x, g)=g v(x)$, where $v: \Delta \longrightarrow \mathbb{R}^{d}$ lies in $F$. Suppose that $m \times \nu$ is ergodic, where $\nu$ is Haar measure on $G$. Let $\phi \in F_{G}$ with $\int_{\Delta \times G} \phi d(m \times \nu)=0$. By Theorem 5.7, we have the following results.

(1) The covariance matrix

$$
\Sigma=\lim _{N \rightarrow \infty}(1 / N) \int_{\Delta \times G} \phi_{N} \phi_{N}^{\mathrm{T}} d(m \times \nu)
$$

exists. Moreover, $\operatorname{det} \Sigma=0$ if and only if there is a nonzero vector $c \in \mathbb{R}^{d}$ such that $c \cdot \phi=c \cdot \psi \circ f-c \cdot \psi$, where $\psi \in F_{G}$.

(2) $\phi_{N}$ satisfies the $d$-dimensional central limit theorem and weak invariance principle on $\Delta \times G$.

(3) If in addition $m \times \nu$ is weak mixing, then we obtain exponential decay of correlations.

6.2.2. Observations on $M$. We now relate the quasicompactness of the equivariant Perron-Frobenius operator on the quotiented tower $\Delta \times G$ to the statistical properties of equivariant observations on $M \times G$.

We define the space $F_{G}$ of $G$-equivariant observations on $M \times G$ by $\phi(x, g)=g v(x)$, where $v: M \longrightarrow \mathbb{R}^{d}$ is Hölder of exponent $\eta$. Let $h: M \longrightarrow G$ lie in the space of Lipschitz cocycles. We form the $G$-extension $\mathcal{T}(x, g)=(\mathcal{F} x, g h(x))$.

We let $\bar{h}$ denote the lift of $h$ to $\bar{\Delta}$ and similarly define $\bar{v}, \bar{\phi}$. Let $\mathcal{B}$ denote the $\sigma$ algebra on $\Delta$ and define $\mathcal{B}_{s}=\left\{\bar{\pi}^{-1} A: A \in \mathcal{B}\right\}$, where $\bar{\pi}: \bar{\Delta} \longrightarrow \Delta$ is the projection. Following Young $[\mathbf{3 9}, \S 5.2]$, we let $h_{0}=E_{\bar{m}}\left(\bar{h} \mid \mathcal{B}_{s}\right)$ denote the conditional expectation of $\bar{h}$ with respect to $\mathcal{B}_{s}$. Similarly, define $v_{0}$ and $\phi_{0}(x, g)=g v_{0}(x)$. This defines $v_{0}, h_{0}$ on $\Delta$ as well, and $\phi_{0}$ on $\Delta \times G$. The assumption that $\beta \geqslant \max \left\{\sqrt{\alpha}, \alpha^{\eta}\right\}$ implies that $v_{0} \in F$ and $\phi_{0} \in F_{G}$. Finally, define $T_{0}(x, g)=\left(f x, g h_{0}(x)\right)$. 
Form the $G$-extension of $(\bar{f}, \bar{\Delta}, \bar{m})$ by defining

$$
\bar{T}(\bar{x}, g)=(\bar{f} \bar{x}, g \bar{h}(\bar{x})) \text {. }
$$

We construct the natural extension of $\bar{T}: \bar{\Delta} \times G \longrightarrow \bar{\Delta} \times G$, with invariant measure $\widehat{m} \times \nu$, and denote it by $\widehat{T}: Y \longrightarrow Y$, where $Y=(\bar{\Delta} \times G)^{\mathbb{Z}^{+}}$. Let $\widehat{\pi}: Y \longrightarrow \bar{\Delta} \times G$ denote the natural projection and let $\widehat{\mathcal{B}}$ denote the $\sigma$-algebra $\left(\widehat{\pi}^{-1} \mathcal{B}_{s}\right) \times \mathcal{B}_{G}$, where $\mathcal{B}_{G}$ is the usual Borel algebra on $G$. Lift $\bar{\phi}$ to $Y$ and denote the lift

$$
\widehat{\phi}(y)=\bar{\phi}(\widehat{\pi} y) \text {. }
$$

To establish the central limit theorem, we apply Gordin [17] as is done in Young [39].

Let

$$
\widehat{\phi}_{j}=E_{\widehat{m}}\left(\widehat{\phi} \mid \widehat{T}^{j} \widehat{\mathcal{B}}\right)
$$

Since we have uniform contraction on stable manifolds by [39, Condition P3],

$$
\left|\widehat{\phi}_{j}-\widehat{\phi}\right| \leqslant C \alpha^{j \eta}
$$

and hence

$$
\sum_{j \geqslant 0}\left|\widehat{\phi}_{j}-\widehat{\phi}\right|_{2}<\infty,
$$

and so the first condition of Gordin's theorem is satisfied.

We now consider $\widehat{\phi}_{-j}$. Since the order of conditioning commutes,

$$
E_{\widehat{m}}\left(\widehat{\phi} \mid \widehat{T}^{-j} \widehat{\mathcal{B}}\right)=E_{m}\left(\phi_{0} \mid T_{0}^{-j} \mathcal{B}\right) \mid,
$$

so it suffices to prove that $\sum_{j \geqslant 0}\left|E_{m}\left(\phi_{0} \mid T_{0}^{-j} \mathcal{B}\right)\right|_{2}<\infty$. This follows immediately from quasicompactness of the equivariant transfer operator, hence proving the central limit theorem. The weak invariance principle follows by standard techniques. Exponential decay of correlations follows as in [39, §4.1] or [1, Proposition 4.2].

Hence we have the following theorem for Hölder $G$-equivariant observations $\phi: M \times G \longrightarrow \mathbb{R}^{d}$ with mean zero and ergodic Lipschitz cocycles $h: M \longrightarrow G$.

(1) The covariance matrix $\Sigma=\lim _{N \rightarrow \infty}(1 / N) \int_{M \times G} \phi_{N} \phi_{N}^{\mathrm{T}} d(\mu \times \nu)$ exists. Moreover, $\operatorname{det} \Sigma=0$ if and only if there is a nonzero vector $c \in \mathbb{R}^{d}$ such that $c \cdot \phi=c \cdot \psi \circ \mathcal{T}-c \cdot \psi$, where $\psi: M \times G \longrightarrow \mathbb{R}^{d}$ is $G$-equivariant and Hölder.

(2) $\phi_{N}$ satisfies the $d$-dimensional central limit theorem and weak invariance principle on $M \times G$.

(3) If in addition $\mu \times \nu$ is weak mixing, then we obtain exponential decay of correlations.

Acknowledgements. We thank Gerhard Keller for very helpful discussions.

\section{References}

1. V. Baladi, Positive transfer operators and decay of correlations, Advanced Series in Nonlinear Dynamics 16 (World Scientific, 2000).

2. V. BAladi, 'Decay of correlations', Smooth ergodic theory and its applications, Proceedings of Symposia in Pure Mathematics 69 (American Mathematical Society, Providence, RI, 2001) 297-325.

3. M. BENEDICKS and L.-S. Young, 'Absolutely continuous invariant measures and random perturbations for certain one-dimensional maps', Ergodic Theory Dynam. Systems 12 (1992) 13-37. 
4. M. Benedicks and L.-S. Young, 'Sinai-Bowen-Ruelle measures for certain Hénon maps', Invent. Math. 112 (1993) 541-576.

5. P. Billingsley, 'The Lindeberg-Lévy theorem for martingales', Proc. Amer. Math. Soc. 12 (1961) 788-792.

6. P. Billingsley, Convergence of probability measures (John Wiley, New York, 1968).

7. P. Billingsley, Probability and measure (John Wiley, New York, 1986).

8. R. Bowen, Equilibrium states and the ergodic theory of Anosov diffeomorphisms, Lecture Notes in Mathematics 470 (Springer, Berlin, 1975).

9. R. Bowen, 'Bernoulli maps of an interval to itself', Israel J. Math. 28 (1978) 298-314.

10. A. BoyARSKY and P. GorA, Laws of chaos: invariant measures and dynamical systems in one dimension, Probability and Its Applications (Birkhäuser, 1997).

11. A. CAstro, 'Backward inducing and exponential decay of correlations for partially hyperbolic attractors with mostly contracting direction,' PhD Thesis, IMPA, 1998.

12. N. Chernov, 'Statistical properties of piecewise smooth hyperbolic systems in high dimensions', Discrete Contin. Dynam. Systems 5 (1999) 425-448.

13. N. Chernov, 'Decay of correlations and dispersing billiards', J. Statist. Phys. 94 (1999) 513-556.

14. J.-P. Conze and S. LE Borgne, 'Méthode de martingales et flow géodésique sur une surface de courbure constante négative', Ergodic Theory Dynam. Systems 21 (2001) 421-441.

15. D. Dolgopyat, 'On dynamics of mostly contracting diffeomorphisms', Comm. Math. Phys. 213 (2000) 181-201.

16. M. J. Field, I. Melbourne and A. TöröK, 'Decay of correlations, central limit theorems and approximation by Brownian motion for compact Lie group extensions', Ergodic Theory Dynam. Systems 23 (2003) 87-110.

17. M. I. Gordin, 'The central limit theorem for stationary processes', Soviet Math. Dokl. 10 (1969) 1174-1176.

18. H. Hennion, 'Sur un théorème spectral et son application aux noyaux lipschitziens', Proc. Amer. Math. Soc. 118 (1993) 627-634.

19. H. Hennion and L. HeRvé, Limit theorems for Markov chains and stochastic properties of dynamical systems by quasi-compactness, Lecture Notes in Mathematics 1766 (Springer, Berlin, 2001).

20. F. Hofbauer, 'On intrinsic ergodicity of piecewise monotonic transformations with positive entropy', Israel J. Math 34 (1979) 213-237.

21. F. Hofbauer, 'On intrinsic ergodicity of piecewise monotonic transformations with positive entropy II', Israel J. Math 38 (1981) 107-115.

22. F. Hofbauer and G. Keller, 'Ergodic properties of invariant measures for piecewise monotonic transformations', Math. Z. 180 (1982) 119-140.

23. G. KELler, 'Piecewise monotonic transformations and exactness', Preprint, 1978.

24. G. Keller and C. Liverani, 'Stability of the spectrum for transfer operators', Ann. Scuola Norm. Sup. Pisa Cl. Sci. XXVIII (1999) 141-152.

25. A. Lasota and J. Yorke, 'On the existence of invariant measures for piecewise monotonic transformations', Trans. Amer. Math. Soc. 186 (1973) 481-488.

26. T. Li and J. Yorke, 'Ergodic transformations from an interval into itself', Trans. Amer. Math. Soc. 235 (1978) 183-192.

27. I. Melbourne and M. Nicol, 'Statistical limit laws for equivariant observations', Stochastics Dynam. 4 (2004) 1-13.

28. M. NiCOL and A. SCOTT, 'Livšic theorems for group extensions of hyperbolic systems with singularities', Ergodic Theory Dynam. Systems 23 (2003) 1867-1889.

29. M. Nicol, I. Melbourne and P. Ashwin, 'Euclidean extensions of dynamical systems', Nonlinearity 14 (2001) 275-300.

30. W. PArry and M. Pollicott, 'The Chebotarev theorem for Galois coverings of Axiom A flows', Ergodic Theory Dynam. Systems 6 (1986) 133-148.

31. W. PARry and M. Pollicott, 'Zeta functions and the periodic orbit structure of hyperbolic dynamics', Astérisque 187-188 (Societé Mathématique de France, Paris, 1990).

32. V. A. Rohlin, 'Exact endomorphisms of a Lebesgue space', Izv. Akad. Nauk SSSR Ser. Mat. 25 (1961) 499-530.

33. D. Ruelle, Thermodynamic formalism, Encyclopedia of Mathematics and its Applications 5 (Addison-Wesley, MA, 1978).

34. S. TAkAhashi, 'Notes on the law of the iterated logarithm', Studia Sci. Math. Hungar. 7 (1972) 21-24.

35. M. VianA, 'Stochastic dynamics of deterministic systems', Col. Bras. de Matemática, 1997.

36. G. WAGNER, 'The ergodic behaviour of piecewise monotonic transformations', $Z$. Wahrscheinlichkeitstheorie und verwandt Gebiete 46 (1979) 317-324. 
37. P. Walters, 'Equilibrium states for $\beta$-transformations and related transformations', Math. Z. 159 (1978) 65-88.

38. S. WONG, 'Some metric properties of piecewise monotonic mappings of the unit interval', Trans. Amer. Math. Soc. 246 (1978) 493-500.

39. L.-S. Young, 'Statistical properties of systems with some hyperbolicity including certain billiards', Ann. of Math. 147 (1998) 585-650.

Ian Melbourne and Matthew Nicol

Department of Mathematics and Statistics

University of Surrey

Guildford

Surrey GU2 7XH

United Kingdom 